\title{
The Effect of Print Angulation on the Accuracy and Precision of 3D-Printed Orthodontic Retainers
}

Allison Williams

Virginia Commonwealth University

Follow this and additional works at: https://scholarscompass.vcu.edu/etd

Part of the Orthodontics and Orthodontology Commons

(C) The Author

\section{Downloaded from}

https://scholarscompass.vcu.edu/etd/6201

This Thesis is brought to you for free and open access by the Graduate School at VCU Scholars Compass. It has been accepted for inclusion in Theses and Dissertations by an authorized administrator of VCU Scholars Compass.

For more information, please contact libcompass@vcu.edu. 
(C) Allison Williams, DDS. 2020.

All Rights Reserved 
The Effect of Print Angulation on the Accuracy and Precision of 3D-Printed Orthodontic Retainers

A thesis submitted in partial fulfillment of the requirements for the degree of Master of Science in Dentistry at Virginia Commonwealth University.

\author{
By \\ Allison Williams \\ Indiana University, BS, 2014 \\ Indiana University, DDS, 2018
}

Thesis advisors:

Eser Tüfekçi, DDS, MS, PhD, MSHA

Professor, Department of Orthodontics

Sompop Bencharit, DDS, MS, PhD

Associate Professor and Director of Digital Dentistry, Department of General Practice

Virginia Commonwealth University

Richmond, Virginia

May, 2020 


\section{Acknowledgements}

Without the support of several individuals, this study would not have been possible. I want to thank my advisor, Dr. Eser Tüfekçi, for guiding me through this process since its genesis. She has been instrumental in helping me work through the logistics in managing a project of this scale and challenging me to grow as a researcher. Dr. Sompop Bencharit taught me how to 3D print and he generously allowed us to use his lab. Dr. Il-Hyung Yang is truly gifted in his ability to understand a multitude of computer programs; his kindness and patience as we toiled through data collection was paramount. Tonya Spangler generously gave her time to be my second rater, and I could not have done this without her help. I want to thank Dr. Caroline Carrico and Spiro Christos Stilianoudakis, my statisticians, for their diligence and understanding on how to best to analyze and share the findings of my study. Finally, none of this would be possible without my husband, Matt Williams. He has been my rock. For all the moments he has given me advice, direction, and encouragement throughout this journey, I am truly grateful. 


\section{Table of Contents}

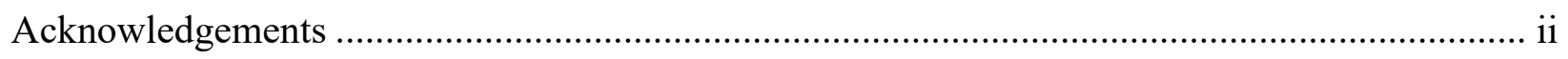

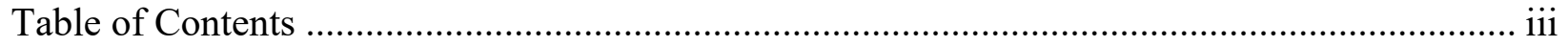

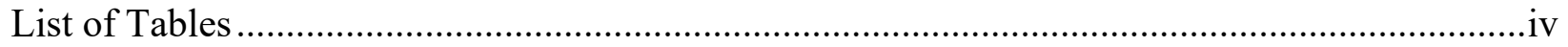

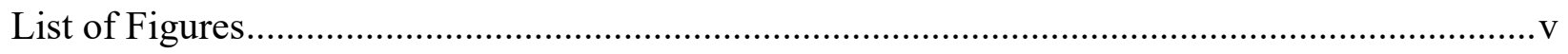

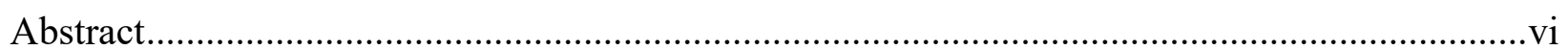

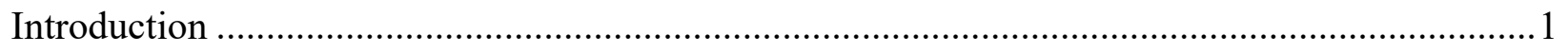

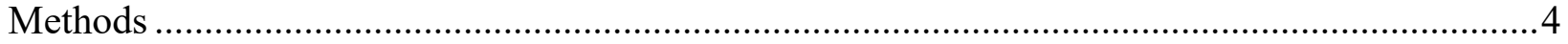

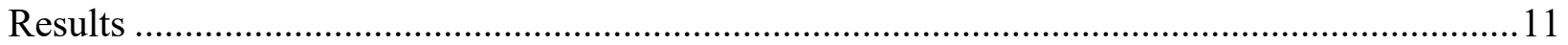

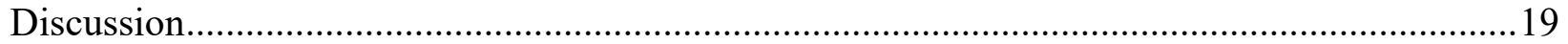

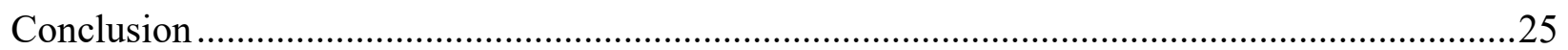

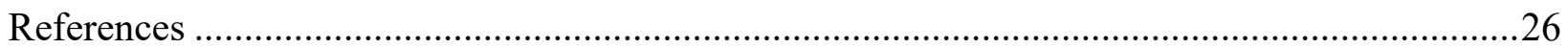




\section{List of Tables}

Table 1. Intraclass Correlation Coefficients between the two raters for each landmark.....11 Table 2. Post-hoc Tukey HSD tests showing the estimated mean differences between Print 1 and Print 2 at each angle and for each landmark.

Table 3. Overall mean discrepancy across landmarks....................................................... 13

Table 4. Mean discrepancy between reference STL file and printed retainers across print angulations.

Table 5. Post-hoc Tukey HSD tests showing the estimated mean discrepancy between printed retainers and the original for each landmark..

Table 6. Comparison of print time, amount of print layers, the volume of resin used, and the cost of the resin. 


\section{List of Figures}

Figure 1. Outline for design of printing process. 60 retainers were printed at five angulations ( $n=12$, each). .5

Figure 2. Layout of retainers at 15 degrees in Preform ${ }^{\mathrm{TM}}$

Figure 3. Layout of retainers at 90 degrees in Preform ${ }^{\mathrm{TM}}$............................................. 7

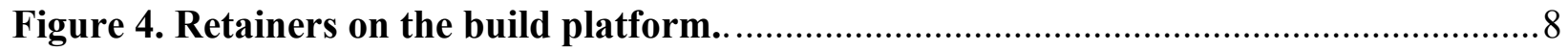

Figure 5. Netfabb ${ }^{\text {TM }}$ “compare meshes" function result..................................................... 9

Figure 6. Mean discrepancy between reference STL file and printed retainers across landmarks......

Figure 7. Mean discrepancy between reference STL file and printed retainers across landmarks.

Figure 8. Bar plots illustrating the mean difference in print accuracy for each landmark, across each print angulation. 


\begin{abstract}
THE EFFECT OF PRINT ANGULATION ON THE ACCURACY AND PRECISION OF 3DPRINTED ORTHODONTIC RETAINERS

By: ALLISON WILLIAMS, DDS

A thesis submitted in partial fulfillment of the requirements for the degree of Master of Science in Dentistry at Virginia Commonwealth University.
\end{abstract}

Virginia Commonwealth University, 2020

Thesis Advisors:

Eser Tüfekçi, DDS, MS, PhD, MSHA

Professor, Department of Orthodontics

Sompop Bencharit, DDS, MS, PhD

Associate Professor and Director of Digital Dentistry, Department of General Practice

Purpose: The aims of this study were: 1) to compare the accuracy and precision of 3-

dimensional (3D) printed retainers at various angulations, 2) to evaluate the effect of angulation on printing time and the amount of resin consumed.

Methods: Using a stereolithography (SLA) 3D printer, a total of 60 clear retainers were printed at five angulations ( $n=12$, each): 15, 30, 45, 60, and 90 degrees. Six retainers were printed each cycle at a random order for all print angulations as print 1 and print 2. Digital images of the original and printed retainers were superimposed. Discrepancies on eight landmarks were measured by two independent examiners. $0.25 \mathrm{~mm}$ was set as the clinically acceptable threshold to determine the accuracy of the retainers.

Results: Deviations ranged from $0.074 \mathrm{~mm}$ to $0.225 \mathrm{~mm}$ from the reference retainer at the cusp tips and incisal edges at all angulations, falling within the threshold of clinical acceptance. Smooth surfaces ranged from $0.263 \mathrm{~mm}$ to $0.480 \mathrm{~mm}$, falling beyond the level of clinical acceptance. Printing at 15 degrees was estimated to be the most time-efficient, while printing at 45 degrees was estimated to be the most cost-effective.

Conclusions: 3D-printed retainers, using an SLA printer, were found to be accurate within 0.25 $\mathrm{mm}$ at all print angulations at the cusp tips and incisal edges when compared to the reference digital file. Smooth facial surfaces fell beyond of the level of clinical acceptability. Printing at 15 degrees was estimated to be the most time-efficient, while printing at 45 degrees was estimated to be the most cost-effective. 


\section{Introduction}

Revolutionized by new technologies, the orthodontic field is transforming to allow for a more digitized clinical workflow. For example, the introduction of intraoral scanners and rapid prototyping with three-dimensional (3D) printers has changed the way orthodontists manage their office. ${ }^{1}$ Instead of taking alginate impressions and pouring up a model in plaster, clinicians can now obtain digital models with intraoral scanners and fabricate 3D-printed appliances, retainers, or indirect bonding trays..$^{2-5}$ As a result, the marriage of the intraoral scanner and 3D printer provides a more effective way to treat and communicate with patients, while also streamlining clinical efficiency. ${ }^{6}$

The accuracy of intraoral scanners has been extensively studied. ${ }^{2}$ In a systematic review, Aragón et $\mathrm{al}^{2}$ reported that intraoral scanners were able to produce accurate and reliable digital models. ${ }^{2}$ Favero et $\mathrm{al}^{7}$ also demonstrated that many commonly used $3 \mathrm{D}$ printer systems reliably produce full-arch dental replicas within $0.25 \mathrm{~mm}$ of the same stone model. The American Board of Orthodontics report discrepancies up to $0.50 \mathrm{~mm}$ as clinically acceptable for the evaluation of a digital articulation using dental models. ${ }^{8}$ However, according to previous studies, disparities as low as 0.15 to $0.25 \mathrm{~mm}$ can result in tooth movement when using clear aligners. ${ }^{9,10}$

Orthodontic retainer fabrication currently relies on reproducing a plaster or 3D-printed model as a first step, followed by a thermoforming process as a second step to create the final appliance. ${ }^{5,11}$ While this method is demonstrably quick and accurate, 3D printing a retainer directly from a digital model would save time and resources. The idea of direct printing was first 
experimented by Nasef et al ${ }^{12,13}$ who designed and fabricated a 3D-printed opaque retainer from a cone-beam computed tomography (CBCT) scan. According to these authors no clinically significant differences were noted between the 3D-printed retainer compared to a conventional vacuum-formed retainer. In a recent study, Cole et $\mathrm{al}^{14}$ found that $3 \mathrm{D}$-printed retainers displayed similar fit, namely at the cusp tips and the incisal edges, to those of the conventional vacuumformed appliances.

Most 3D printing manufacturers recommend printing dental models at an angulation below 30 degrees to yield the highest precision, smoothness, and accuracy. ${ }^{11,15}$ To efficiently produce the greatest number of models, the printing needs to be oriented as close to vertical as possible. On most 3D printing build platforms, using an angulation below 30 degrees allows only a maximum of six retainers to be printed at one time. Adjusting the print angulation to 90 degrees provides space for 12 retainers. Doubling production may potentially increase clinical efficiency; however, no studies thus far have explored this outcome in retainers. In a study by Short et al, ${ }^{15}$ dental models printed in multiple orientations, including vertically, were within $0.25 \mathrm{~mm}$ exhibiting clinical acceptability.

Printing clear retainers at an angle may cause a risk of distortion or inaccuracy because of the transparency of the resin. As part of the manufacturing process of most $3 \mathrm{D}$ printers, the photopolymer resin is exposed to a laser of a certain wavelength, followed by an ultraviolet light cure. ${ }^{6,16-20}$ The transparency of the material used for 3D printing may overexpose some parts of the appliance to the laser during the additive building process as light travels through. This phenomenon may affect the final accuracy of clear retainer specifically through an overbuilding, or addition of too much photopolymer resin, to certain areas of the retainer. ${ }^{20}$ 
Print angulations may also affect printing time and the amount of resin used.,15 Currently, there is no information on the time- and cost-effectiveness of $3 \mathrm{D}$ printing when different print orientations are used. Furthermore, a change in print angulation and its subsequent impact on the overall accuracy and precision of 3D-printed orthodontic retainers remain unclear. Therefore, the aims of this study were: 1) to compare the accuracy and precision of 3D-printed clear retainers at various angle setting, 2) to evaluate the effect of angulation on printing time and the amount of consumed resin. 


\section{Methods}

In this study, a previously created standard tessellation language (STL) file of a clear orthodontic retainer, with a thickness of $0.75 \mathrm{~mm}$, was used to fabricate the 3D-printed

retainers. ${ }^{14}$ Retainers were printed with modifications made to the original STL file that included reference landmarks on selected areas. These landmarks served to eliminate error during the superimposition process which will be discussed in detail later.

Using a stereolithography (SLA) 3D printer (Form 2; Formlabs, Somerville, MA) and a clear resin, (Clear, FormLabs Inc; Somerville, MA) a total of 60 maxillary retainers were printed at five print angulations ( $\mathrm{n}=12$, each): 15, 30 45, 60, and 90 degrees. Print angulations of 15 and 30 degrees served as controls based on the manufacturer's recommended angulation for printing clear dental splints. ${ }^{11}$ Currently, there is not a specific recommended print angulation for fabricating clear retainers; therefore, due to the similarity of the resin and appliance type, the printing methodology for clear dental splints was followed. The study design is outlined in Figure 1. Because the build platform for the Form 2 3D printer is 145 x $145 \mathrm{~mm}$ in dimension, only six retainers can be printed at 15 and 30 degrees of angulation (Figure 2). ${ }^{11}$ Even though more than six retainers can be fabricated at one printing undertaking with 45, 60 and 90 degrees of angulation (Figure 3), to control for confounding variables, only six retainers were printed each cycle at a random order for all five print angulations as print 1 and print 2 . 


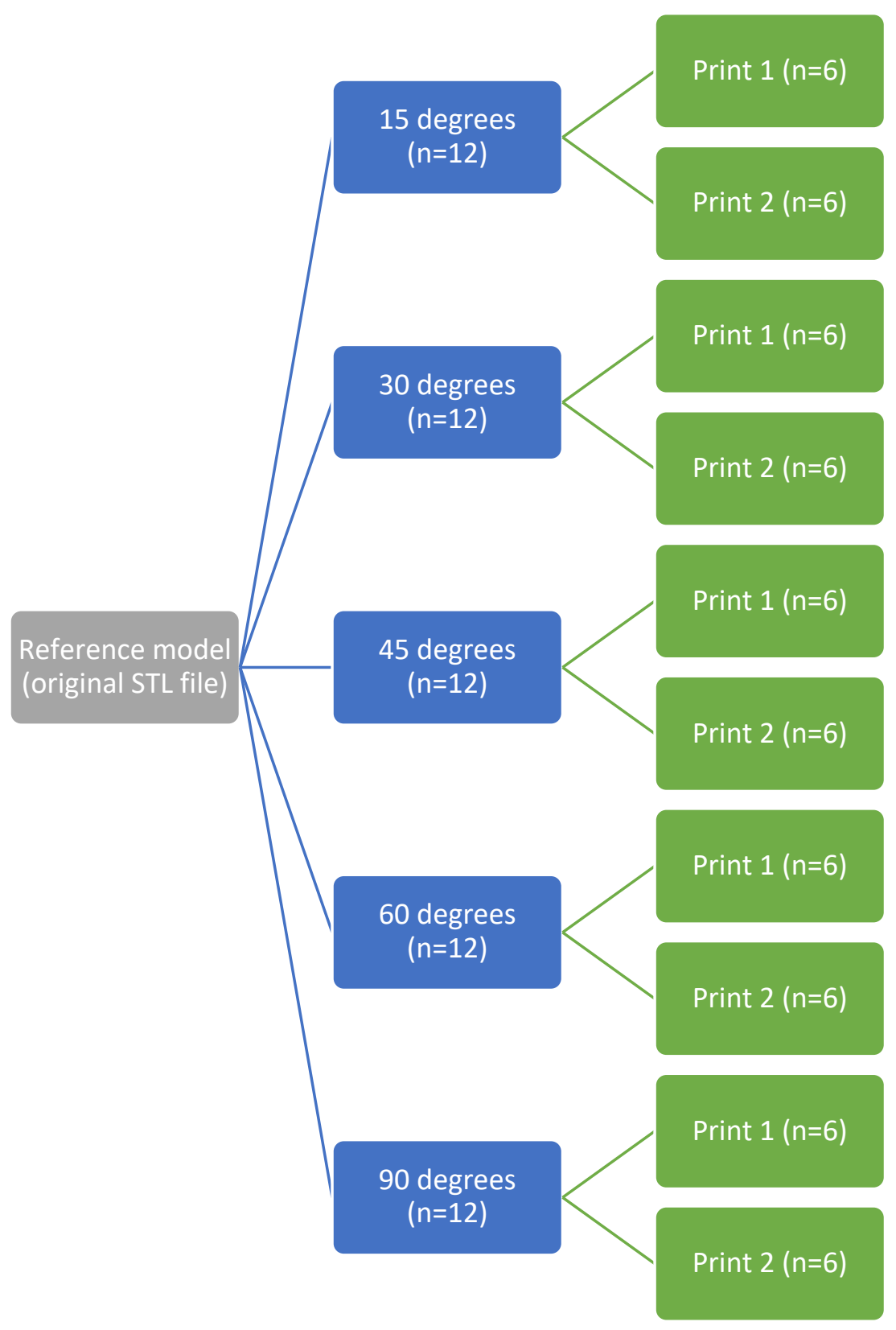

Figure 1. Outline for design of printing process. 60 retainers were printed at five angulations $(\mathbf{n}=\mathbf{1 2}$, each). For the standardization purposes, retainers were printed in batches (print 1 and print 2) of six per print at all angulations. 


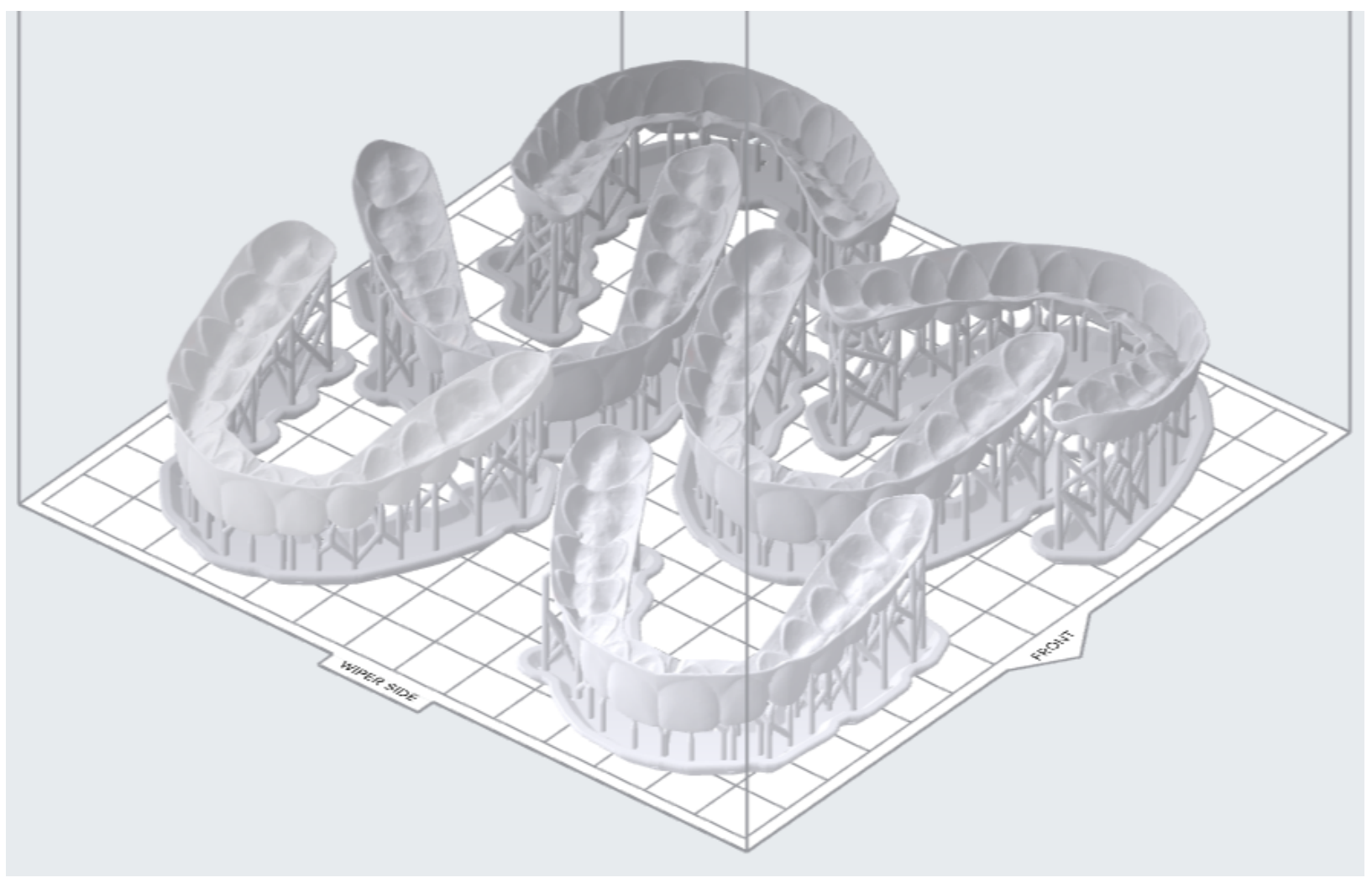

Figure 2. Layout of retainers at 15 degrees in Preform ${ }^{\mathrm{TM}}$. 


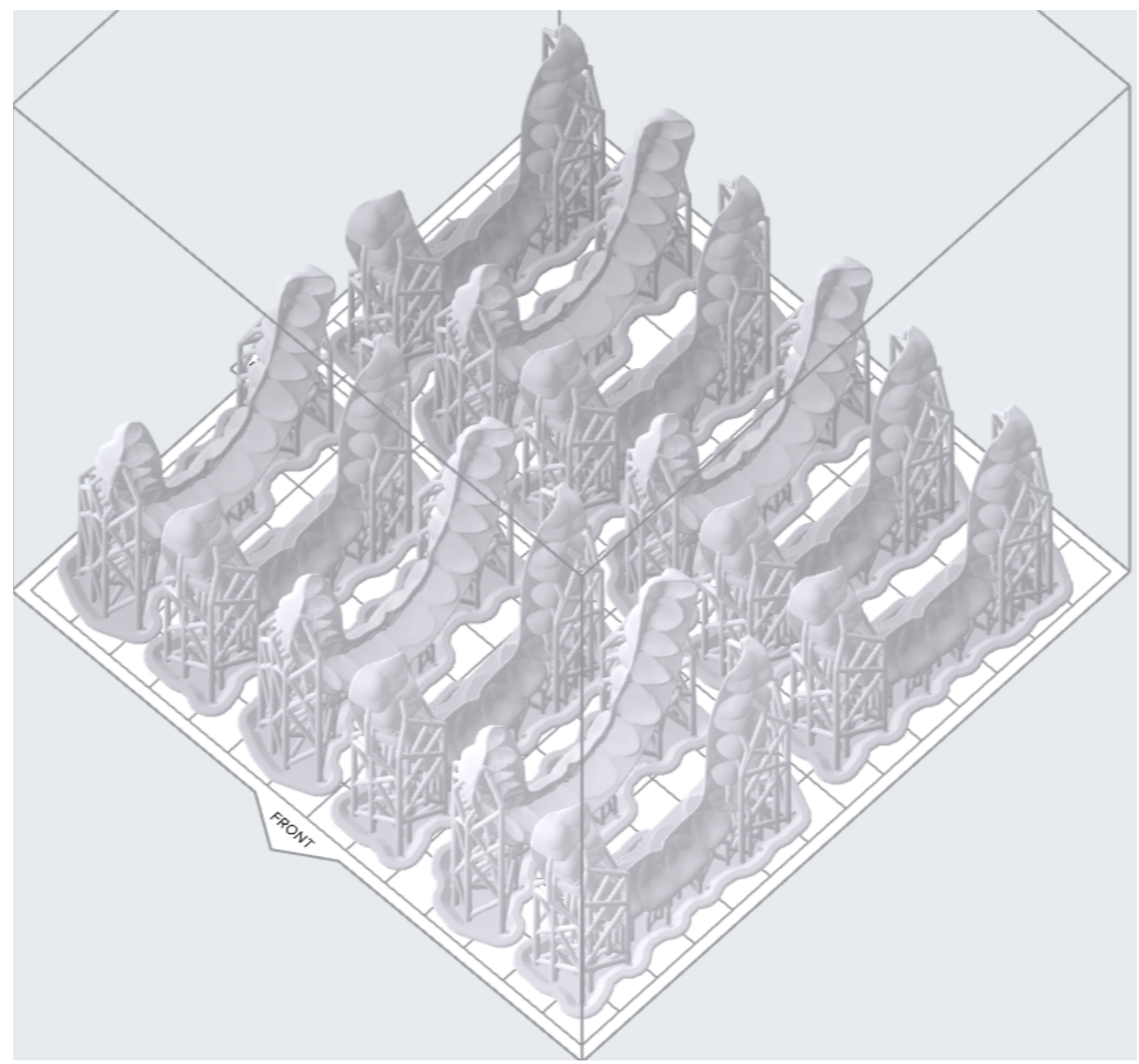

Figure 3. Layout of retainers at 90 degrees in Preform ${ }^{\mathrm{TM}}$. The Form 2 build platform can accommodate up to 12 retainers.

During printing process, the STL file of the retainer was imported into PreForm ${ }^{\mathrm{TM}}$, the software used for the Form 2 SLA 3D printer. The retainers were then printed with a clear resin at a resolution of $100 \mu \mathrm{m}$, and subsequently were subjected to a post-processing treatment. Retainers were first rinsed in 96\% isopropyl alcohol for 5 minutes and then cured for 30 minutes 
with an ultraviolet light at $60^{\circ} \mathrm{C}$, as per the FormLabs manufacturing instructions for fabricating a dental splint. ${ }^{11}$

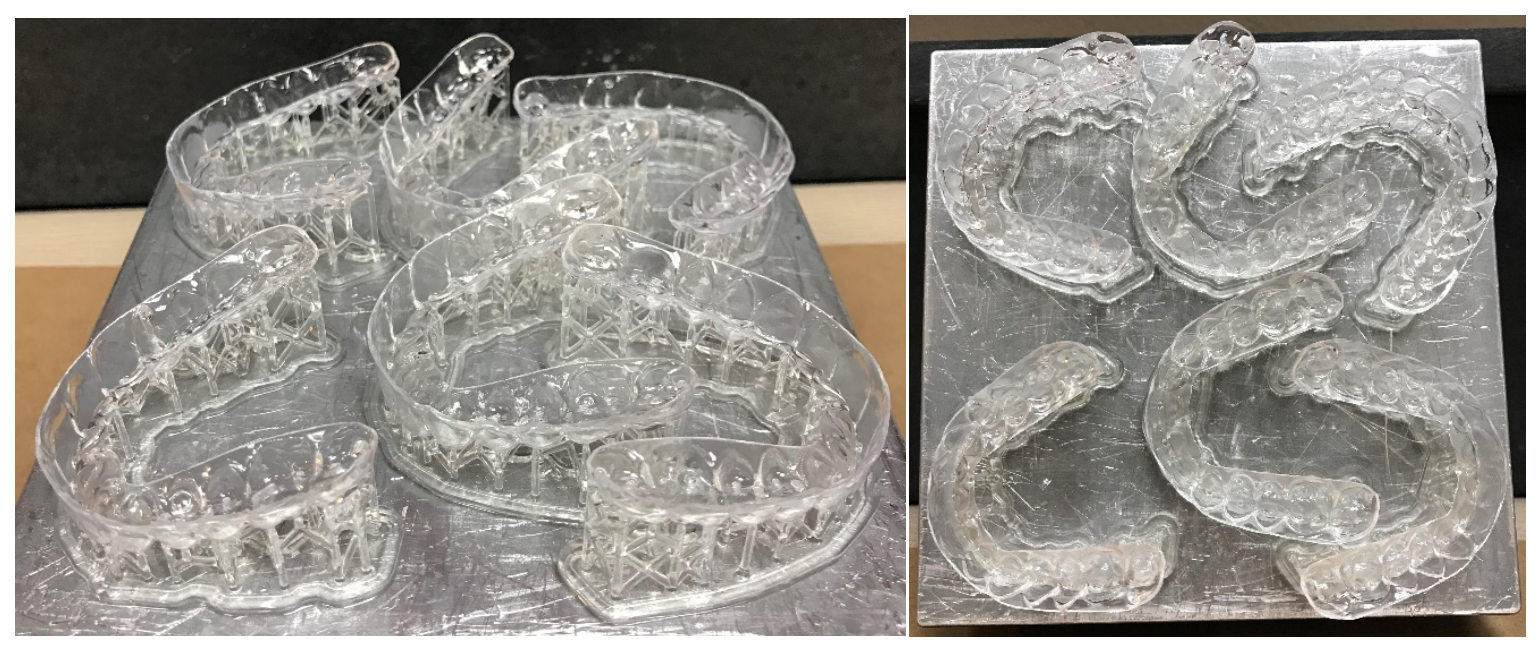

Figure 4. Retainers on the build platform. A total of 6 retainers were printed for each print batch. This is an example of retainers printed at 15 degrees.

After post-processing the retainers, a $\mathrm{CBCT}$ scan, that has been previously deemed reliable for reproducing the intricacies the dentition, ${ }^{21}$ was performed. The images of the printed retainers were then converted into an STL file using Invesalius ${ }^{\mathrm{TM}}$ (Centro de Tecnologia da Informação Renato Archer; Campinas, Brazil). The STL files were modified to remove the supports. This would typically be included in a post-processing step; however, it was completed virtually to reduce human error. Next, the files were imported into a 3D analyzing software, Netfabb (Netfabb; Autodesk Inc., San Rafael, CA).

To aid with the superimposition process, reference points were previously added to the intaglio surface of the digital image with the aid of Meshmixer ${ }^{\mathrm{TM}}$ software (Autodesk Inc.; San Rafael, CA). Ball markers (1.5 mm in diameter), serving as landmarks for an accurate superimposition, were placed at the mesiobuccal cusp of the right and left first molars (R6 and L6, respectively), the cusp tip of the right and left canine (R3 and L3, respectively), the middle 
of the incisal edge of the right and left central incisors (R1 and L1, respectively), and the right and left central incisor mid-facial points (FR1 and FL1, respectively). The retainer was digitally made symmetrical to assess the reliability of the print between the right and left sides.

The original and the newly scanned STL files for each retainer (60 total) were superimposed by two independent examiners using the "compare meshes" function in Netfabb ${ }^{\mathrm{TM}}$ (Figure 5). Deviations between the original and the 3D-printed retainers were computed from the superimpositions using the analytical features of the software.

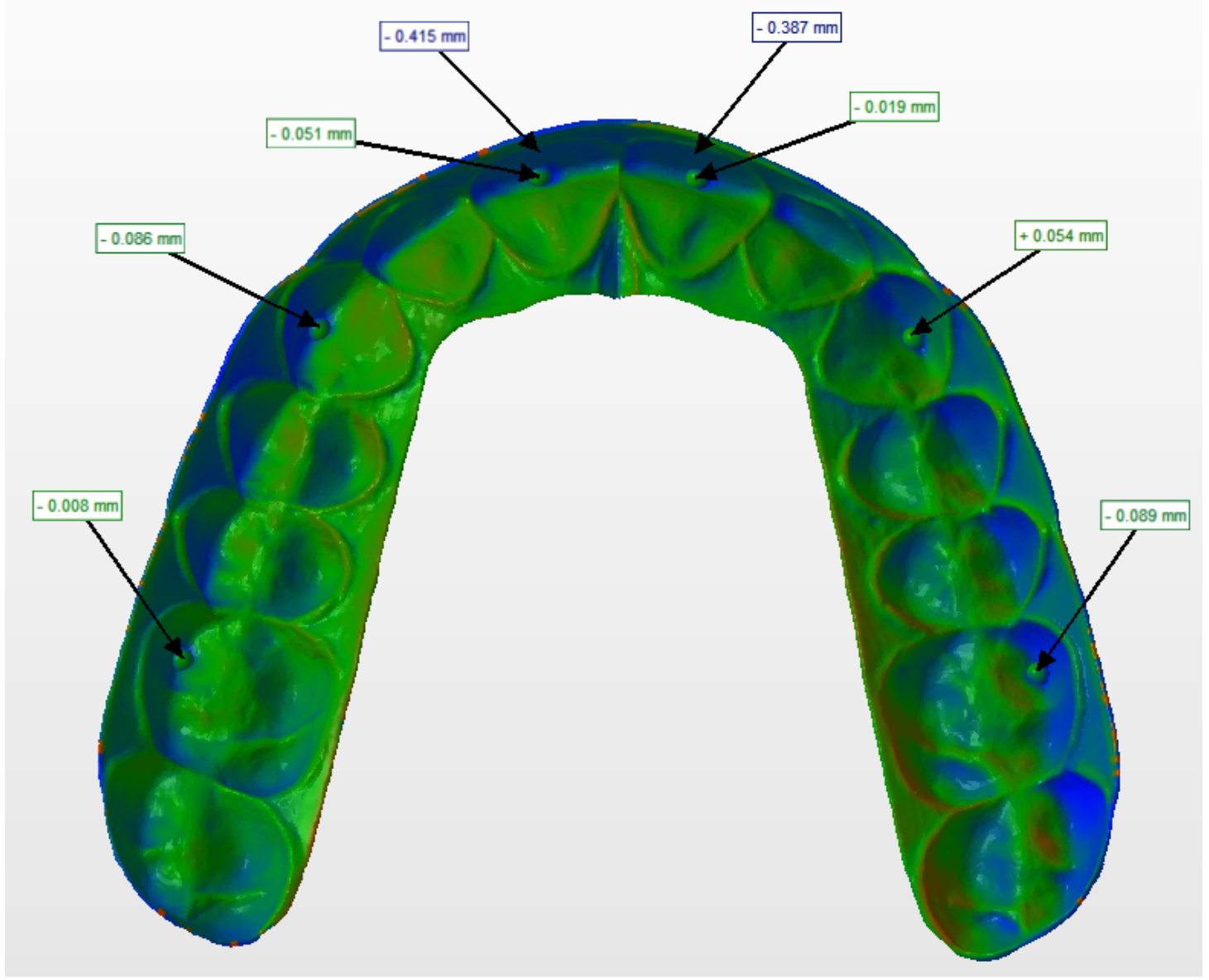

Figure 5. Netfabb "compare meshes" function result. Measurements were made at the first molars, canines, and central incisors (R6, L6, R3, L3, R1, L1, FR1, FL1). 
These discrepancies have been compiled for data analyses for accuracy and precision. For the secondary aims, the printing time and the amount of consumed resin were recorded for each print angulation.

\section{STATISTICAL ANALYSES}

Agreement in assessing the differences between the STL images of the original retainer and each of the printed retainers at the eight reference points was determined using Intraclass Correlation Coefficients (ICC). Differences for the print angulations and print batches were assessed using a one-way and two-way analysis of variance (ANOVA) by comparing the measurements at reference points on the STL images of the original and printed retainers. Posthoc pairwise comparisons were adjusted using Tukey Honestly Significant Difference (HSD). All statistical analyses were performed in R (version 3.6.1) (R Development Core Team; University of Auckland, New Zealand). The significance level was kept at $\leq 0.05$ alpha level of significance. 


\section{Results}

The intraclass correlation coefficients between the two raters for each landmark are provided in Table 1. Results indicated that the agreement in the assessments between the two raters at each landmark ranged from $0.446(\mathrm{p}=0.0001)$ at the $\mathrm{R} 6$ landmark to $0.967(\mathrm{p}<0.0001)$ at the FL1 landmark. The differences in the measurement values between the two raters were negligible; therefore, the percent agreement within $0.10 \mathrm{~mm}$ was used to further evaluate the agreement (Table 1). At all landmarks, measurements were within $0.10 \mathrm{~mm}$ at least $85 \%$ of the time. Deviations up to $0.10 \mathrm{~mm}$ between examiners were found statistically not significant and all subsequent analyses were performed using the maximum difference value between the measurements of two raters.

Table 1. Intraclass Correlation Coefficients between the two raters for each landmark.

\begin{tabular}{|c|c|c|c|}
\hline Landmark & ICC (95\% CI) & $\begin{array}{c}\text { Percentage with } \\
<\mathbf{0 . 1 0} \mathbf{~ m m ~ d i f f e r e n c e ~}\end{array}$ & P-Value \\
\hline R6 & $0.446(0.221,0.626)$ & $93.3 \%$ & 0.0001 \\
\hline L6 & $0.955(0.926,0.973)$ & $100.0 \%$ & $<0.0001$ \\
\hline R3 & $0.815(0.700,0.887)$ & $91.7 \%$ & $<0.0001$ \\
\hline L3 & $0.794(0.678,0.872)$ & $86.7 \%$ & $<0.0001$ \\
\hline R1 & $0.860(0.776,0.914)$ & $90.0 \%$ & $<0.0001$ \\
\hline L1 & $0.733(0.536,0.845)$ & $85.0 \%$ & $<0.0001$ \\
\hline FR1 & $0.960(0.855,0.983)$ & $100.0 \%$ & $<0.0001$ \\
\hline FL1 & $0.967(0.945,0.980)$ & $100.0 \%$ & $<0.0001$ \\
\hline
\end{tabular}

There were statistically significant differences between the print 1 and print 2 retainers for each of the eight landmarks according to post-hoc Tukey HSD Tests (Table 2). However, the largest difference between print batches was $0.278 \mathrm{~mm}$, and only two out of the 40 measurements (8 landmarks at 5 print angulations) exceeded the $0.25 \mathrm{~mm}$ threshold. After 
empirically evaluating measurements between print 1 and print 2 batches, the retainers in the two groups were combined for further analyses since the differences were not clinically significant.

Table 2. Post-hoc Tukey HSD tests showing the estimated mean differences between Print 1 and Print 2 at each angle and for each landmark.

\begin{tabular}{|c|c|c|c|c|c|}
\hline Landmark & $\begin{array}{c}\text { Angulation } \\
\text { (degrees) }\end{array}$ & Print 1 & Print 2 & $\begin{array}{l}\text { Estimated Mean } \\
\text { Print Difference }\end{array}$ & P-Value \\
\hline \multirow[t]{5}{*}{ R6 } & 15 & 0.085 & 0.156 & -0.070 & 0.0133 \\
\hline & 30 & 0.092 & 0.135 & -0.043 & 0.115 \\
\hline & 45 & 0.096 & 0.141 & -0.045 & 0.102 \\
\hline & 60 & 0.079 & 0.094 & -0.014 & 0.5938 \\
\hline & 90 & 0.089 & 0.127 & -0.037 & 0.1712 \\
\hline \multirow[t]{5}{*}{ L6 } & 15 & 0.108 & 0.094 & 0.013 & 0.7292 \\
\hline & 30 & 0.247 & 0.075 & 0.172 & 0.0001 \\
\hline & 45 & 0.058 & 0.337 & -0.278 & $<.0001$ \\
\hline & 60 & 0.106 & 0.041 & 0.065 & 0.1003 \\
\hline & 90 & 0.094 & 0.072 & 0.022 & 0.5601 \\
\hline \multirow[t]{5}{*}{$\mathbf{R 3}$} & 15 & 0.083 & 0.101 & -0.017 & 0.6554 \\
\hline & 30 & 0.165 & 0.063 & 0.102 & 0.0093 \\
\hline & 45 & 0.094 & 0.258 & -0.164 & 0.0001 \\
\hline & 60 & 0.131 & 0.115 & 0.015 & 0.6866 \\
\hline & 90 & 0.239 & 0.210 & 0.029 & 0.436 \\
\hline \multirow[t]{5}{*}{ L3 } & 15 & 0.105 & 0.138 & -0.033 & 0.509 \\
\hline & 30 & 0.243 & 0.114 & 0.128 & 0.0126 \\
\hline & 45 & 0.108 & 0.281 & -0.172 & 0.0011 \\
\hline & 60 & 0.244 & 0.132 & 0.112 & 0.0278 \\
\hline & 90 & 0.128 & 0.089 & 0.039 & 0.4331 \\
\hline \multirow[t]{5}{*}{ R1 } & 15 & 0.108 & 0.096 & 0.011 & 0.7842 \\
\hline & 30 & 0.231 & 0.093 & 0.138 & 0.0017 \\
\hline & 45 & 0.128 & 0.312 & -0.183 & 0.0001 \\
\hline & 60 & 0.103 & 0.068 & 0.034 & 0.4113 \\
\hline & 90 & 0.138 & 0.092 & 0.045 & 0.2763 \\
\hline \multirow[t]{5}{*}{ L1 } & 15 & 0.131 & 0.136 & -0.004 & 0.9053 \\
\hline & 30 & 0.239 & 0.097 & 0.142 & 0.0006 \\
\hline & 45 & 0.087 & 0.280 & -0.193 & $<.0001$ \\
\hline & 60 & 0.149 & 0.078 & 0.071 & 0.0742 \\
\hline & 90 & 0.227 & 0.194 & 0.033 & 0.3994 \\
\hline \multirow[t]{4}{*}{ FR1 } & 15 & 0.342 & 0.380 & -0.037 & 0.465 \\
\hline & 30 & 0.200 & 0.325 & -0.125 & 0.0173 \\
\hline & 45 & 0.546 & 0.293 & 0.253 & $<.0001$ \\
\hline & 60 & 0.265 & 0.325 & -0.060 & 0.2404 \\
\hline
\end{tabular}




\begin{tabular}{|c|c|c|c|c|c|}
\hline & 90 & 0.305 & 0.447 & -0.142 & 0.0073 \\
\hline FL1 & 15 & 0.341 & 0.414 & -0.072 & 0.2514 \\
\hline & 30 & 0.289 & 0.399 & -0.110 & 0.0817 \\
\hline & 45 & 0.585 & 0.374 & 0.211 & 0.0013 \\
\hline & 60 & 0.366 & 0.389 & -0.022 & 0.7218 \\
\hline & 90 & 0.295 & 0.522 & -0.226 & 0.0006 \\
\hline
\end{tabular}

The overall mean discrepancies of the landmarks between the reference STL file and the printed retainers calculated from Table 3 were $0.111 \mathrm{~mm}$ at $\mathrm{R} 6,0.124 \mathrm{~mm}$ at $\mathrm{L} 6,0.147 \mathrm{~mm}$ at R3, $0.159 \mathrm{~mm}$ at $\mathrm{L} 3,0.137 \mathrm{~mm}$ at R1, $0.162 \mathrm{~mm}$ at L1, $0.343 \mathrm{~mm}$ at FR1, and $0.399 \mathrm{~mm}$ at FL1. Only measurements at the smooth surfaces of the central incisors (FR1, FL1) were beyond 0.25 $\mathrm{mm}$, the threshold for clinical acceptance. Mean discrepancies are illustrated in Figure 6.

Table 3. Overall mean discrepancy across landmarks.

\begin{tabular}{|c|c|c|}
\hline Landmark & Mean Difference (mm) & Standard Deviation \\
\hline R6 & 0.111 & 0.051 \\
\hline L6 & 0.124 & 0.109 \\
\hline R3 & 0.147 & 0.089 \\
\hline L3 & 0.159 & 0.103 \\
\hline R1 & 0.137 & 0.099 \\
\hline L1 & 0.162 & 0.091 \\
\hline FR1 & 0.343 & 0.123 \\
\hline FL1 & 0.399 & 0.133 \\
\hline
\end{tabular}




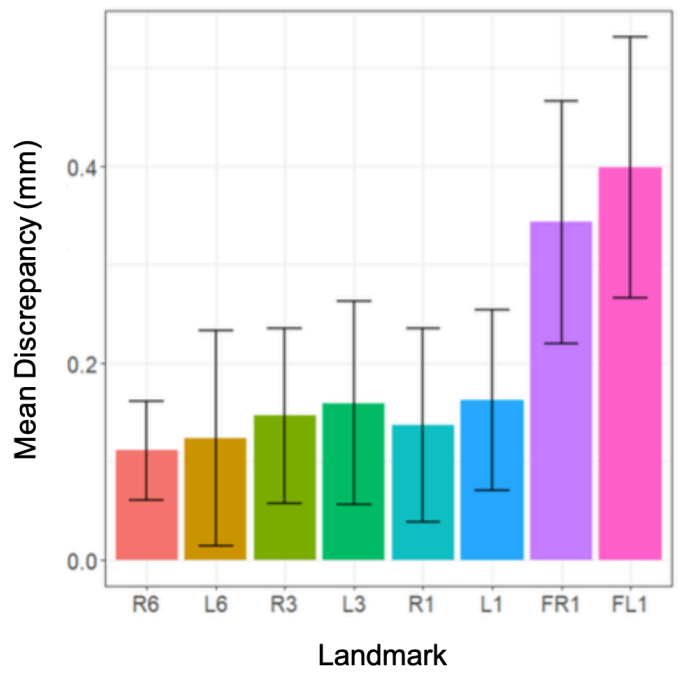

Figure 6. Mean discrepancy between reference STL file and printed retainers across landmarks.

The overall mean discrepancies between the reference STL file and the printed retainers across print angulations are shown in Table 4 . The differences were $0.179 \mathrm{~mm}$ at 15 degrees, $0.188 \mathrm{~mm}$ at 30 degrees, $0.249 \mathrm{~mm}$ at 45 degrees, $0.168 \mathrm{~mm}$ at 60 degrees, and $0.205 \mathrm{~mm}$ at 90 degrees. Mean discrepancies are illustrated in Figure 7.

Table 4. Mean discrepancy between reference STL file and printed retainers across print angulations.

\begin{tabular}{|c|cc|}
\hline Angulation (degrees) & Mean Difference $\mathbf{( m m )}$ & Standard Deviation \\
\hline $\mathbf{1 5}$ & 0.179 & 0.139 \\
\hline $\mathbf{3 0}$ & 0.188 & 0.120 \\
\hline $\mathbf{4 5}$ & 0.249 & 0.165 \\
$\mathbf{6 0}$ & 0.168 & 0.122 \\
\hline $\mathbf{9 0}$ & 0.205 & 0.158 \\
\hline
\end{tabular}




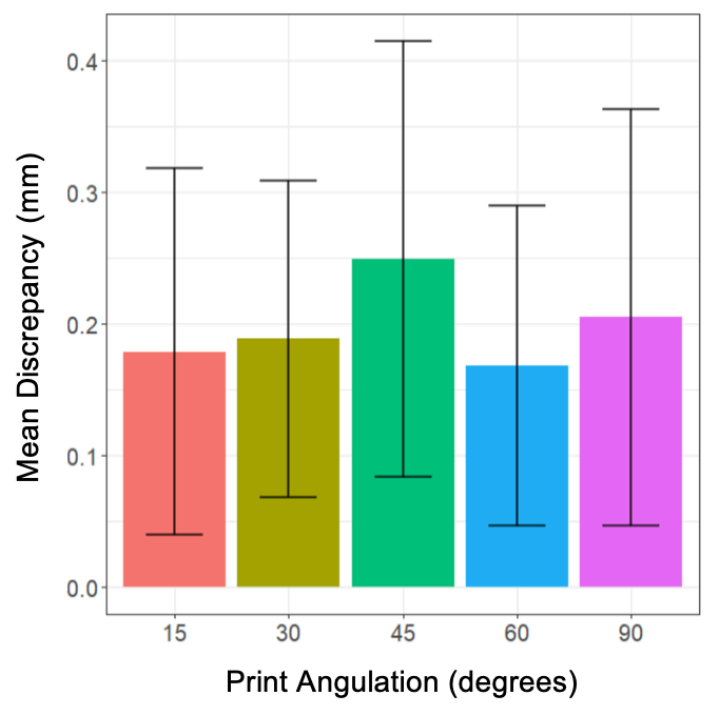

Figure 7. Mean discrepancy between reference STL file and printed retainers across landmarks.

Table 5 displays the estimated mean discrepancy between the reference STL file and the 3D-printed retainers across landmarks and print angulations. The R6, L3, L1, and FL1 landmarks did not present statistically significant estimated mean differences between print angulations whereas the L6, R3, R1, and FR1 landmarks did present with statistically significant estimated mean differences. When comparing between print angulations, the largest and the most frequently occurring statistically meaningful difference was seen at the 45-degree angle print, with L6, R1, and FR1 values exhibiting at least one statistically significant pairwise difference. Within each print angulation, differences at FR1 and FL1 landmarks were much larger than the differences presented at the other landmarks (mean difference $=0.343 \mathrm{~mm}$ and $0.399 \mathrm{~mm}$, respectively). 
Table 5. Post-hoc Tukey HSD tests showing the estimated mean discrepancy between printed retainers and the original for each landmark. P-values correspond to the overall oneway ANOVA tests. The angulations with the same letter indicate that they are not significantly different from each other.

\begin{tabular}{|c|c|c|c|c|}
\hline Landmark & $\begin{array}{l}\text { Print Angulation } \\
\text { (degrees) }\end{array}$ & $\begin{array}{c}\text { Estimated Mean } \\
\text { Discrepancy }(\mathrm{mm})\end{array}$ & $\begin{array}{l}\text { Standard } \\
\text { Error }\end{array}$ & P-Value \\
\hline \multirow[t]{6}{*}{ R6 } & & & & 0.3882 \\
\hline & 15 & 0.127 & 0.0146 & $\mathrm{a}$ \\
\hline & 30 & 0.114 & 0.0146 & $\mathrm{a}$ \\
\hline & 45 & 0.119 & 0.0146 & $\mathrm{a}$ \\
\hline & 60 & 0.087 & 0.0146 & $\mathrm{a}$ \\
\hline & 90 & 0.109 & 0.0146 & $\mathrm{a}$ \\
\hline \multirow[t]{6}{*}{ L6 } & & & & 0.01501 \\
\hline & 15 & 0.101 & 0.0292 & $a b$ \\
\hline & 30 & 0.162 & 0.0292 & $a b$ \\
\hline & 45 & 0.198 & 0.0292 & $\mathrm{~b}$ \\
\hline & 60 & 0.074 & 0.0292 & $\mathrm{a}$ \\
\hline & 90 & 0.083 & 0.0292 & $a b$ \\
\hline \multirow[t]{6}{*}{$\mathbf{R 3}$} & & & & 0.000661 \\
\hline & 15 & 0.094 & 0.0224 & $\mathrm{a}$ \\
\hline & 30 & 0.114 & 0.0224 & $\mathrm{a}$ \\
\hline & 45 & 0.177 & 0.0224 & $\mathrm{ab}$ \\
\hline & 60 & 0.124 & 0.0224 & $\mathrm{a}$ \\
\hline & 90 & 0.225 & 0.0224 & $\mathrm{~b}$ \\
\hline \multirow[t]{6}{*}{ L3 } & & & & 0.1292 \\
\hline & 15 & 0.124 & 0.0289 & $\mathrm{a}$ \\
\hline & 30 & 0.179 & 0.0289 & $\mathrm{a}$ \\
\hline & 45 & 0.195 & 0.0289 & $\mathrm{a}$ \\
\hline & 60 & 0.189 & 0.0289 & $\mathrm{a}$ \\
\hline & 90 & 0.109 & 0.0289 & $\mathrm{a}$ \\
\hline \multirow[t]{6}{*}{ R1 } & & & & 0.002831 \\
\hline & 15 & 0.101 & 0.0255 & $\mathrm{a}$ \\
\hline & 30 & 0.162 & 0.0255 & $a b$ \\
\hline & 45 & 0.220 & 0.0255 & $\mathrm{~b}$ \\
\hline & 60 & 0.086 & 0.0255 & $\mathrm{a}$ \\
\hline & 90 & 0.115 & 0.0255 & $\mathrm{a}$ \\
\hline \multirow[t]{6}{*}{ L1 } & & & & 0.06535 \\
\hline & 15 & 0.134 & 0.0252 & $\mathrm{a}$ \\
\hline & 30 & 0.168 & 0.0252 & $\mathrm{a}$ \\
\hline & 45 & 0.183 & 0.0252 & $\mathrm{a}$ \\
\hline & 60 & 0.114 & 0.0252 & $\mathrm{a}$ \\
\hline & 90 & 0.211 & 0.0252 & $\mathrm{a}$ \\
\hline FR1 & & & & 0.008537 \\
\hline
\end{tabular}




\begin{tabular}{|c|c|c|c|c|}
\hline & 15 & 0.364 & 0.0326 & $\mathrm{ab}$ \\
\hline & 30 & 0.263 & 0.0326 & $\mathrm{a}$ \\
\hline & 45 & 0.419 & 0.0326 & $\mathrm{~b}$ \\
\hline $\mathbf{F L 1}$ & 60 & 0.295 & 0.0326 & $\mathrm{ab}$ \\
\hline & 90 & 0.376 & 0.0326 & $\mathrm{ab}$ \\
\hline & 15 & & & $\mathbf{0 . 1 3 0 5}$ \\
\hline & 30 & 0.384 & 0.0373 & $\mathrm{a}$ \\
\hline & 45 & 0.344 & 0.0373 & $\mathrm{a}$ \\
\hline & 60 & 0.480 & 0.0373 & $\mathrm{a}$ \\
\hline & 90 & 0.378 & 0.0373 & $\mathrm{a}$ \\
\hline
\end{tabular}

The mean differences in print accuracy for each landmark across each print angulation is provided in Figure 8. All of the landmarks except the FR1 and FL1 are below the clinically acceptable threshold of $0.25 \mathrm{~mm}$, which is indicated by the dashed line.

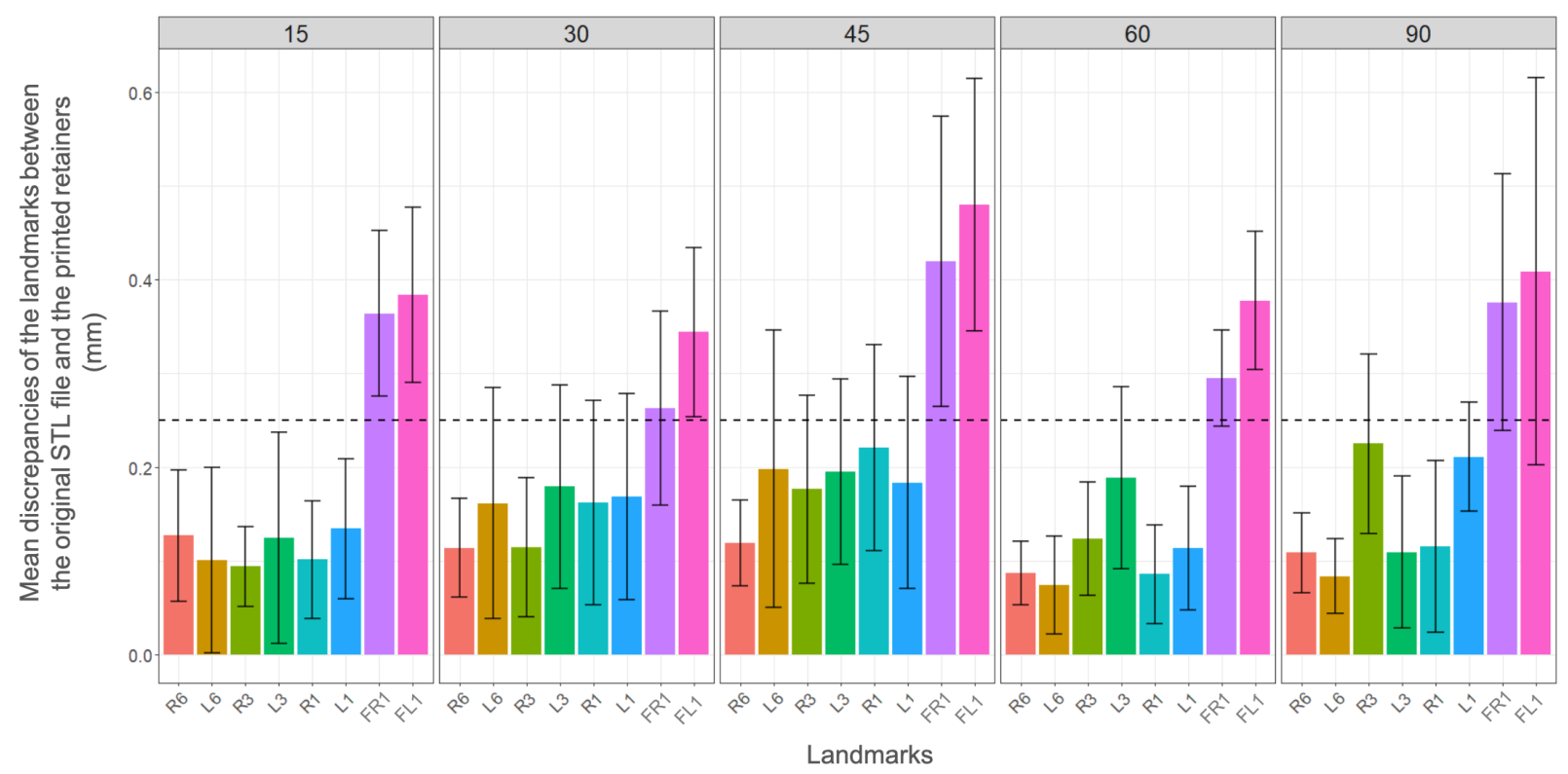

Figure 8. Bar plots illustrating the mean difference in print accuracy for each landmark, across each print angulation. The error bars represent one standard deviation away from the mean difference. 
For the secondary aims, printing time, number of print layers, and volume of resin were compared (Table 6). Values were estimated based on printing one retainer. Printing one retainer at 15 degrees of angulation took the least amount of time at 1 hour and 30 minutes while printing at 60 and 90 degrees lasted for 2 hours and 15 minutes. The total amount of resin used per retainer (in volume) is provided in Table 6. Forty-five degrees was the most cost-effective print angulation consuming $5.20 \mathrm{~mL}$ of resin per retainer. Printing at 30 degrees resulted in the most amount of resin consumed, and therefore would yield the costliest retainer among the five experimental print angulations.

Table 6. Comparison of estimated print time, amount of print layers, and the volume of resin used. These values represent the data for printing one retainer. The values increase for each variable if more than one retainer is printed.

\begin{tabular}{|c|c|c|c|}
\hline $\begin{array}{c}\text { Print Angulation } \\
\text { (degrees) }\end{array}$ & Print Time & Print Layers & Volume of resin $(\mathrm{mL})$ \\
\hline 15 & $1 \mathrm{hr} 30 \mathrm{~min}$ & 286 & 5.33 \\
\hline 30 & $2 \mathrm{hr}$ & 391 & 6.40 \\
\hline 45 & $2 \mathrm{hr}$ & 477 & 5.20 \\
\hline 60 & $2 \mathrm{hr} 15 \mathrm{~min}$ & 537 & 5.57 \\
\hline 90 & $2 \mathrm{hr} 15 \mathrm{~min}$ & 573 & 6.22 \\
\hline
\end{tabular}




\section{Discussion}

In recent years, orthodontics has welcomed digital technology offering advantages over conventional methods that improve treatment diagnostics and clinical efficiency. Specifically, the intraoral scanner, an important tool eagerly adopted into the orthodontic practice, has remained pivotal in treatment planning and record-keeping. ${ }^{2,6}$ Today, intraoral scanners can be paired with $3 \mathrm{D}$ printers to directly fabricate clear retainers and aligners. ${ }^{6}$ Traditionally, clear retainers and other appliances are fabricated with a two-step approach using either a plaster or a 3D-printed cast. With advances in technology, digitally created casts may be used to print the appliances in just one step. To date, there is limited information on the fit of 3D-printed clear orthodontic retainers, and ongoing research continues to integrate various technologies to improve the practice of orthodontics. ${ }^{14}$

To print clear appliances, such as dental splints, 3D printing manufacturers recommend a print angulation of 30 degrees or less, a print setup that can only fit six retainers per platform. The angulation may be altered to increase the number of appliances fabricated in one print cycle. Studies have investigated the effect of print angulation on the accuracy and precision of 3Dprinted dental models; however, this information is not available for retainers. ${ }^{15-17,22,23}$ Therefore, in this study the print angulations were modified to investigate the effects on quality assurance of deviation from the suggested print angulation. 
Previous studies utilized a superimposition technique to determine the accuracy and precision of 3D-printed retainers by comparing the digitally created image of the appliance to its original STL file. ${ }^{12,14}$ Prior research has demonstrated that using a spray to scan the intaglio surfaces of the retainers added a significant amount of material thickness to the samples during the process. ${ }^{14}$ In this study, to negate this error the retainers were scanned with CBCT. ${ }^{21}$ Also, pre-determined eight landmarks were used for the superimposition of the STL files when assessing accuracy and precision. ${ }^{14,16}$

When grading plaster models, the American Board of Orthodontics considers any difference under $0.5 \mathrm{~mm}$ as clinically acceptable. ${ }^{8}$ However, for a retainer to maintain tooth position a closer adaptation may be clinically necessary. According to Boyd and Vlaskalic ${ }^{9}$ for an aligner to act as an active appliance a minimum of 0.15 to $0.25 \mathrm{~mm}$ distance needs to exist between the plaster model and the appliance. Therefore, in this study differences up to $0.25 \mathrm{~mm}$ were considered as clinically acceptable for retention, and statistical analyses were performed based on this assumption..$^{9,10}$

Printer reliability and precision was analyzed based on the difference between print batches. A total of 12 retainers per angulation were printed in two batches, each containing six retainers. Clinical acceptance was set at $0.25 \mathrm{~mm}$. Only two out of the 40 measurements exhibited differences between print batches greater than $0.25 \mathrm{~mm}$. The two landmarks measured $0.253 \mathrm{~mm}$ and $0.278 \mathrm{~mm}$ different between print batch 1 and print batch 2 . Both of the landmarks were found in the 45-degree print angulation group. Discrepancies between print batches may have been caused by an error in post-processing, data collection, or printer inaccuracy. 
Overall, the reference STL file and the digital image of the printed retainers demonstrated negligible discrepancies at the cusp tips and incisal edges with an average range between 0.074 $\mathrm{mm}$ and $0.225 \mathrm{~mm}$. Meanwhile, smooth facial surface landmarks at the central incisors provided the greatest observed differences with an average range between $0.263 \mathrm{~mm}$ and $0.480 \mathrm{~mm}$. Cole et al ${ }^{14}$ showed similar findings suggesting that the printing capabilities may be limited in these areas. ${ }^{14}$ To assess whether using a more robust 3D analysis software would have an effect on the findings, four randomly selected retainers were also evaluated with Geomagic (3D Systems, Inc., Rock Hill, SC). The color map generated by Geomagic showed that the discrepancies between the reference and 3D-printed retainer files were similar. Therefore, in this study while statistically significant differences were noted in most landmarks, the differences at or below $0.25 \mathrm{~mm}$ were treated as no clinical significance other than at the smooth surfaces of central incisors. Future studies to evaluate the fit of the retainers at the smooth surfaces of teeth on original models or in patients are therefore warranted.

According to Formlabs, the manufacturer of the 3D printer used in this study, printing 1.0 $\mathrm{mm}$ thick dental splints at an angulation greater than 30 degrees are expected to result in an inaccurate replication of interproximal surfaces. ${ }^{11}$ However, the warning against printing at angulations lower than 30 degrees may not apply for retainers that typically measure 0.50 to 0.75 $\mathrm{mm}$ in thickness. Based on the results of this study, there does not appear to be clinically significant difference between print angulations less than 30 degrees compared to steeper angulations. This finding may enhance the clinical workflow of retainer fabrication in the orthodontic practice, allowing orthodontists to $3 \mathrm{D}$ print a greater number of retainers in one print batch. 
Currently, there is not an approved resin commercially available for direct printing of clear orthodontic retainers. The clear resin chosen for this study has the ability to be printed at a resolution as low as $25 \mu \mathrm{m}$ while maintaining transparent properties. The accuracy of a future resin designed for intraoral retainer use may have the capability to print at lower resolutions, perhaps improving accuracy, precision, and ultimately fit of the retainer. Currently, a clear biocompatible resin (Dental LT Clear, FormLabs Inc; Somerville, MA) only supports a print resolution of $100 \mu \mathrm{m}$. In 2018, a 3D printing company and leader in digital technology, EnvisionTEC (EnvisionTEC, Inc; Dearborn, MI), announced the beginning stages of production of an ideal resin suitable for direct printing of retainers. ${ }^{19}$ The current study attempts to establish a clinical recommendation by testing the accuracy and precision of retainers printed on an SLA printer with an available resin in the market. Based on the results of this study, there does not appear to be a distinct optimal print angulation. However, future studies should implement a variety of printers with different technologies to ascertain whether differences exist among manufacturers.

Based on the absence of significant effect of print angulation on the accuracy, this study suggests that providers may carry out printing tasks based on time or cost efficiency. Printing retainers at an angulation of 90 degrees arranges space for 12 retainers at one time. Meanwhile, 45- and 60-degree angulations yield eight retainers per print cycle with 15 and 30 degrees producing only six retainers. Overall, printing at 30 degrees appears to be the least cost-effective option, consuming the greatest amount of resin per retainer, and printing at 45 degrees is the most cost-effective. The difference in cost stems from the amount of print layers and lattice support structure necessary. Therefore, orthodontists who want to fabricate the greatest number 
of retainers at one print may select the print angulation of 90 degrees while those seeking costeffective options may find printing at 45 degrees preferable.

As previously stated, resins for the direct fabrication of retainers are not yet currently available. In this study, the best commercially available resin was used for the fabrication of the clear retainers due to its transparency after post-processing. The current research has only addressed the effect of the print angulation on the accuracy and precision of clear orthodontic retainers when using an SLA 3D printer.

The SLA printer used in the current study uses additive manufacturing technology to create objects in a layer by layer fashion. Because the laser cures only a small area at a time, the process is highly precise, yet slow. In the current study, printing one retainer ranged from 1 hour and $30 \mathrm{~min}$ to 2 hours and 15 minutes. Therefore, it is possible that using printers with different technologies may result in faster printing times than an SLA printer.

A digital light processing (DLP) printer is another widely-used 3D printer in the orthodontic office. This printer utilizes vat-polymerization, similar to SLA printer, in that the build platform is lowered into the pool of resin while receiving a light cure. Unlike the SLA printer, the DLP printer cures an entire layer at one time with the help of thousands of small mirrors. This rapid light curing process of the DLP printer has been likened to a "stamp" rather than "drawing" each layer like the SLA printer. ${ }^{6}$ The stamping technique allows the DLP printer to produce an object in much less time. Another 3D printing technology, polyjet photopolymerization (PPP), operates by flowing resin onto the surface followed by an immediate ultraviolet light cure. Multiple jets are streamed simultaneously opening the ability of PPP printers to incorporate various materials in the object. ${ }^{6}$ 
Future studies with an approved resin material will be useful for evaluating the mechanical and physical properties along with the potential for clinical applications. In addition, another limitation of the current study was that the retainers were compared to a digital retainer file. Currently, the clear retainers are fabricated manually using the thermoforming method. It would be ideal to compare a 3D-printed retainer to a standard lab-made appliance. Therefore, future studies comparing the 3D-printed retainers to thermoformed retainers are warranted. Similarly, the amount of consumed resin per retainer along with the precision and accuracy need to be investigated in future studies using other printing technologies. 


\section{Conclusions}

1) Retainers fabricated at print angulations of $15,30,45,60$, and 90 degrees were found to be accurate within $0.25 \mathrm{~mm}$ at the canine and molar cusp tips along with the central incisor incisal edges when compared to the reference STL file.

2) Canine and molar cusp tips along with central incisor incisal edges showed the greatest accuracy with a mean range between $0.074 \mathrm{~mm}$ and $0.225 \mathrm{~mm}$ while central incisor smooth facial surfaces exhibited the least accuracy with a mean range between $0.263 \mathrm{~mm}$ and $0.480 \mathrm{~mm}$.

3) The 15-degree print angulation was estimated to be the most time-efficient setting and the 45-degree print angulation was estimated to be the most cost-effective setting. 


\section{References}

1. Sun LJ, Lee JS, Choo HH, Hwang HS, Lee KM. Reproducibility of an intraoral scanner: A comparison between in-vivo and ex-vivo scans. Am J Orthod Dentofac Orthop. 2018;154(2):305-310. doi:10.1016/j.ajodo.2017.09.022

2. Aragón MLC, Pontes LF, Bichara LM, Flores-Mir C, Normando D. Validity and reliability of intraoral scanners compared to conventional gypsum models measurements: a systematic reviewo Title. Eur J Orthod. 2016;38(4):429-434.

3. Taneva E, Kusnoto B, Evans CA. 3D Scanning, Imaging, and Printing in Orthodontics. Issues Comtemporary Orthod. 2015;(Figure 1):147-188. doi:10.5772/60010

4. Retrouvey J, Kader E, Caron E, Tamimi F, Light N. Printing Orthodontic Retainers Using CAD / CAM Technology. 2013;(11):1-5.

5. Mohd Tahir N, Wan Hassan WN, Saub R. Comparing retainers constructed on conventional stone models and on 3D printed models: a randomized crossover clinical study. Eur J Orthod. 2018;02866617:1-11. doi:10.1093/ejo/cjy063

6. Groth C, Kravitz ND, Jones PE, Graham JE, Redmond W. OVERVIEW ThreeDimensional Printing Technology. J Clin Orthod. 2014;48(8):475-485.

7. Favero CS, English JD, Cozad BE, Wirthlin JO, Short MM, Kasper FK. Effect of print layer height and printer type on the accuracy of 3-dimensional printed orthodontic models. Am J Orthod Dentofac Orthop. 2017;152(4):557-565. doi:10.1016/j.ajodo.2017.06.012

8. Sweeney S, Smith DK, Messersmith M. Comparison of 5 types of interocclusal recording materials on the accuracy of articulation of digital models. Am J Orthod Dentofac Orthop. 2015;148(2):245-252. doi:10.1016/j.ajodo.2015.04.025

9. Boyd RL, Waskalic V. Three-dimensional diagnosis and orthodontic treatment of complex malocclusions with the invisalign appliance. Semin Orthod. 2001;7(4):274-293.

doi:10.1053/sodo.2001.25414

10. Phan X, Ling PH. Clinical limitations of invisalign. J Can Dent Assoc (Tor). 2007;73(3):263-266.

11. Formlabs. Manufacturing Thermoformed Clear Aligners and Retainers on 3D Printed Models With the Form 2. 2018;(August).

12. Nasef AA, El-Beialy AR, Eid FHK, Mostafa YA. Accuracy of Orthodontic 3D Printed Retainers versus Thermoformed Retainers. Open J Med Imaging. 2017;07(04):169-179. doi:10.4236/ojmi.2017.74017

13. Nasef AA, El-Beialy AR, Mostafa YA. Virtual techniques for designing and fabricating a retainer. Am J Orthod Dentofac Orthop. 2014;146(3):394-398.

doi:10.1016/j.ajodo.2014.01.025

14. Cole D, Bencharit S, Carrico CK, Arias A, Tüfekçi E. Evaluation of fit for 3D-printed retainers compared with thermoform retainers. Am J Orthod Dentofac Orthop. 2019;155(4):592-599. doi:10.1016/j.ajodo.2018.09.011

15. Short MM, Favero CS, English JD, Kasper FK. Impact of Orientation on Dimensional Accuracy of 3D-Printed Orthodontic Models. J Clin Orthod. 2018;42(1):13-20. 
doi:10.1080/01639360802634134

16. Kim SY, Shin YS, Jung HD, Hwang CJ, Baik HS, Cha JY. Precision and trueness of dental models manufactured with different 3-dimensional printing techniques. Am J Orthod Dentofac Orthop. 2018;153(1):144-153. doi:https://doi.org/10.1016/j.ajodo.2017.05.025

17. Brown GB, Currier GF, Kadioglu O, Kierl JP. Accuracy of 3-dimensional printed dental models reconstructed from digital intraoral impressions. Am J Orthod Dentofac Orthop. 2018;154(5):733-739. doi:10.1016/j.ajodo.2018.06.009

18. Camardella LT, Vilella O V, van Hezel MM, Breuning KH. Accuracy of stereolithographically printed digital models compared to plaster models. J Orofac Orthop. 2017;78(5):394-402. https://link-springercom.proxy.library.vcu.edu/article/10.1007\%2Fs00056-017-0093-1.

19. EnvisionTEC Launches Two Orthodontic Materials at LMT Lab Day Chicago, Including Resin for Direct 3D Printing of Clear Aligners. BusinessWire. https://envisiontec.com/orthodontic-materials-launched-at-lmt-lab-day-chicago/. Published 2018.

20. EnvisionTEC. Understanding 3D Printer Accuracy. 2017. https://envisiontec.com/wpcontent/uploads/2017/08/Accuracy-versus-Resolution-0824172.pdf.

21. Wiranto MG, Engelbrecht WP, Tutein Nolthenius HE, Van Der Meer WJ, Ren Y. Validity, reliability, and reproducibility of linear measurements on digital models obtained from intraoral and cone-beam computed tomography scans of alginate impressions. $\mathrm{Am} \mathrm{J}$ Orthod Dentofac Orthop. 2013;143(1):140-147. doi:10.1016/j.ajodo.2012.06.018

22. Lee KY, Cho JW, Chang NY, et al. Accuracy of three-dimensional printing for manufacturing replica teeth. Korean J Orthod. 2015;45(5):217-225. doi: $10.4041 /$ kjod.2015.45.5.217

23. Jin S, Jeon I, Kim J, Kim W. Accuracy (trueness and precision) of dental models fabricated using additive manufacturing methods. Int J Comput Dent. 2018;21(2):107-113. 